http://jmscr.igmpublication.org/home/ ISSN (e)-2347-176x ISSN (p) 2455-0450

crossref DOI: https://dx.doi.org/10.18535/jmscr/v8i3.32

\author{
Journal Of Medical Science And Clinical Research \\ IGM Publication \\ An official Publication of IGM Publication
}

\title{
A prospective study to compare conventional radiotherapy verses accelerated radiotherapy with concurrent chemotherapy in squamous cell carcinoma of oral cavity
}

Authors

\author{
Anand Prakash Sachan ${ }^{1}$, S.N.Prasad ${ }^{2}$
}

${ }^{1}$ Resident, ${ }^{2}$ Professor and Head J.K.C I G.S.V.M. Medical College, Kanpur, Uttar Pradesh, India

\begin{abstract}
Background: about 1.93 lakhs new cases of head neck cancer are diagnosed and 1.14 lakhs deaths occurred due to head neck cancer, per year in India. India contributes to up to $15.6 \%$ of the global cancer burden and $12.1 \%$ of global cancer deaths. (globocan 2018) ${ }^{[1]}$ India accounts for the highest incidence of oral and oro-pharyngeal cancers. For early stages, chemo-radiotherapy or surgery are equally effective. For advanced stages require multimodality treatment. Standard chemo-radiotherapy requires $2 g y$ per fractions, 5 fractions per week for 7 weeks.

Materials and Methods: Total 57patients (29 for arm a-conventional chemo-radiation and 28 for arm baccelerated radiotherapy) were selected from the cross section of patients registered at the $j . K$. Cancer institute and other associated hospitals of g. S. V. M medical college, Kanpur from December 2017 to august 2019. Histologically proven carcinoma patients by way of biopsy were evaluated. The data thus obtained were assessed, analyzed and compared to find out difference in all the groups in terms of tumor response and quality of life by using $t$ test.

Results: Out of 29 patients, in arm a, 19 (65.52\%) and in arm b, 17 patients (60.71\%) had complete response (cr) and the rest of the patients had partial response.

Conclusion: Accelerated fractionated radiotherapy that is six fractions per week is reasonable alternative to the conventional regimen that is five fractions per week, offers advantages of decreased overall treatment time, better compliance and decreased hospital stay with comparable response rate but significantly increased low grade acute reaction which were manageable.

Keywords: accelerated radiotherapy, chemo-radiotherapy, mucositis, oral cavity, oropharyngeal cancers.
\end{abstract}

\section{Introduction}

Head and neck cancer is the 7th most common type of cancer and $8^{\text {th }}$ most common cancer related death in the world, more than 8 lakhs new cases of head and neck cancer are diagnosed each year. In india head neck cancer is the most common cancer in men, about 1.93 lakhs new cases of head neck cancer are diagnosed and 1.14 lakhs deaths occurred due to head neck cancer, per year in india. India contributes to up to $15.6 \%$ of the global cancer burden and $12.1 \%$ of global cancer deaths. (globocan 2018) ${ }^{[1]}$ india accounts for the highest incidence of lip and oral cancer in the world with over 1,00,000 cases registered annually. ${ }^{[2]}$ chemo-radiotherapy has been identified as a standard therapeutic method in patients with locally advanced squamous cell carcinoma of the head and neck. ${ }^{[3-4]}$ work of maciejewski $^{[5]}$ and withers ${ }^{[6]}$, showed that with increasing overall time the total dose to cure a 
tumour of the head and neck area had to be raised, this was attributed to repopulation, which may not be important until the third week of a course of treatment. Hypofractionated regimens with shorten the overall duration of treatment were therefore investigated with the aim of reducing the time in which cellular repopulation could occur. Several randomised clinical trials have shown an increase in local control using accelerated or hyperfractionated radiotherapy. ${ }^{[7-10]}$ a metaanalysis showed that altered radiotherapy with new fractionating schedules, achieved an increase of $7 \%$ in local control and $3 \%$ in survival at 5 years. $^{[1]}$ accelerating the radiation schedule involves shortening the overall duration of therapy to less than the 7 weeks used in a conventional schedule. The key element is the reduction in the overall time. One problem with a fractionated course of radiation is that tumor regeneration can occur during a course of treatment, reducing the probability of cure. Keane and colleagues calculated that a $2.7 \%$ reduction in local control for patients with tonsillar carcinoma would be seen with each day of treatment prolongation. ${ }^{[12]}$ two randomized trials, in denmark ${ }^{[13]}$ and poland, ${ }^{[14]}$ evaluated conventional therapy with five fractions per week, compared to accelerated regimens using six to seven fractions per week. Total dose and fractionsize remained the same, resulting in a shortening of treatment time by 1 or 2 weeks. In vancouver, canada, jackson and associates attempted a greater reduction in overall treatment time, delivering 66 gy in 33 fractions in either 45 to 48 days or 22 to 25 days. ${ }^{[15]}$

One of the most important biological factors related to the outcome of $\mathrm{rt}$ in squamous cell carcinoma of the head and neck is the proliferation of tumour stem cells during treatment. A cause of resistance with conventional fractionation $\mathrm{rt}$ could be radiation-induced accelerated proliferation of clonogenic tumour cells. A reduction in the chance of tumour control through the lengthening of treatment times has been clinically and biologically documented. ${ }^{[16]}$ furthermore, in a substantial number of clinical reports, reduction in the total treatment time has improved tumour control. ${ }^{[17,18,19]}$ a shorter treatment time can be accomplished by applying a higher dose per fraction, but this change will disproportionately increase the rate of late complications. Accelerated treatment is therefore only possible if the weekly number of fractions is increased without increasing the dose per fraction. This shortening of overall treatment time should limit the extent of accelerated repopulation and therefore one may expect an increase in the probability of tumour control for given total dose. $^{[20]}$ since treatment time is thought to have little or no influence on the response of late reacting normal tissue, a reduction in overall treatment time would not be expected to affect the incidence and severity of late normal tissue injury. At the same time, reducing overall treatment time will increase the turnover on machine, thus will reduce the waiting list also, especially in a busy department like ours with limited resources. Keeping above facts in mind, we have planned a randomized clinical trial to test the efficacy of shortening the overall treatment time from 7 weeks to 6 weeks by delivering six fractions per week instead of five fractions per week in the treatment of head and neck cancers in our institution with the aim to find out whether shortening of overall treatment time by use of this regimen is tolerable and improves the tumour response.

\section{Materials and Methods}

The case material for the study were selected from the cross section of patients registered at the j. K. Cancer institute of G. S. V. M medical college, Kanpur from December 2017 to august 2019. The eligibility criteria included histologically proven squamous cell carcinoma of oral cavity by way of biopsy were evaluated.

Patients accrued for study underwent pretreatment evaluation which included complete history, physical examination and complete systemic examination. Patients were assessed their general 
Condition by kps and bsa. Their hematological assessment was done by complete hemogram,

Biochemical assessment of kidney and liver function, radiological assessment. Dental assessment and care. Patients were staged according to ajcc staging system.

Based on the above assessment the patients for the study were selected depending on histologically proven cases of carcinoma, karnofsky performance status > 70, early and locally advanced oral cavity cancer. Complete hemogram with $\mathrm{hb}>10 \mathrm{gm} / \mathrm{dl}$; tlc- 4000 to $11000 / \mathrm{cmm}$, platelet count $>100,000 / \mathrm{cmm}$. Renal function tests with blood urea $<40 \mathrm{mg} / \mathrm{dl}$ and serum creatinine $<$ $1.5 \mathrm{mg} / \mathrm{dl}$. Liver function tests with sgot $<35 \mathrm{iu} / \mathrm{l}$ and sgpt $<40 \mathrm{iu} / \mathrm{l}$. Patients who sign the informed consent and are ready to be on follow up as required the patients having any of the following conditions were excluded from the study: prior radiation, surgery or chemotherapy for the disease, poor general condition with karnofsky performance status of $<70$, pregnant or lactating patient, associated medical condition such as renal disease, liver disease or heart disease and thus the patients fulfilling the inclusion criteria and exclusion criteria were randomized into two arms as followed:

Arm a: conventional:- 70 gy/35\# with 2 gy / fraction for 5 days / week for 7 weeks with concurrent cisplatin $100 \mathrm{mg} / \mathrm{m} 2$ on d, d22

Arm b: accelerated:-70 gy/35\# with 2 gy / fraction for 6 days / week for 5 weeks 5 days with concurrent cisplatin $100 \mathrm{mg} / \mathrm{m} 2$ on d1, d22.

From the commencement of treatment, all the patients included in the study were carefully and Regularly assessed weekly during treatment. Radiation reactions were assessed by radiation Therapy oncology group (rtog) criteria. Tumor response (both primary and nodal response) were assessed by recist (1.1) response criteria 2 months after completion of radiotherapy.

The major study endpoints were tumor response, acute and late toxicities and quality of life using University of Washington quality of life.
Questionnaire version 4.0 all the patients were assessed two weeks after the completion of treatment, to detect acute complications like mucositis, skin reaction, late reactions based on rtog criteria patients were followed monthly upto a minimum of 6 months . Tumor response was assessed based on recist response criteria1.1 all the patients were followed up regularly on opd basis for a period of at least 6 months, once every month after completion of the treatment. At every visit, each patient was clinically evaluated for local control of disease and treatment related complications. The patients were assessed for any evidence of distant metastasis during each follow up. On suspicion of any local recurrence, biopsy were taken for histopathology and correlated clinically. The QOL were assessed at the beginning of treatment, on the day of completion of treatment and one month after completion of planned treatment using university of Washington QOL questionnaire. The data thus obtained was assessed, analyzed and compared to find out difference in all the groups in terms of tumor response and quality of life by using student $t$ test.

\section{Results}

Total number of patients identified for the trial based on inclusion and exclusion criteria were Randomized to arm a and arm b. All patients in both arm completed the assigned treatment.

Table 1: shows number of patients in each arm

\begin{tabular}{|l|c|c|c|}
\hline Parameter & & $\begin{array}{c}\text { Control arm } \\
(29)\end{array}$ & $\begin{array}{c}\text { Test arm } \\
(28)\end{array}$ \\
\hline Sex & Male & 21 & 23 \\
\cline { 2 - 4 } & Female & 8 & 5 \\
\hline $\begin{array}{l}\text { Most common age } \\
\text { group }\end{array}$ & & $31-50 \mathrm{yrs}$ & $31-50 \mathrm{yrs}$ \\
\hline Residence & Rural & 13 & 16 \\
\cline { 2 - 4 } & Urban & 16 & 12 \\
\hline Site & Tongue & 12 & 9 \\
\cline { 2 - 4 } & $\begin{array}{c}\text { Buccal } \\
\text { mucosa }\end{array}$ & 10 & 10 \\
\cline { 2 - 4 } & Rmt & 2 & 1 \\
\cline { 2 - 4 } & Alveolous & 1 & 1 \\
\cline { 2 - 4 } & Gbs & 1 & 1 \\
\cline { 2 - 4 } & Hard palate & 2 & 5 \\
\cline { 2 - 4 } & Multiple site & 1 & 5 \\
\cline { 2 - 4 } & 2 & 4 & $42-55$ days \\
\cline { 2 - 4 } & 3 & 2 & \\
\hline \multirow{3}{*}{\begin{tabular}{l} 
Stage \\
\cline { 2 - 4 }
\end{tabular}} & 4 & $50-67$ days & \\
\hline \multirow{2}{*}{$\begin{array}{l}\text { Average duration } \\
\text { of treatment }\end{array}$} & & & 7 \\
\hline
\end{tabular}




\section{JMSCR Vol||08||Issue||03||Page 190-197||March}

In our study age wise distribution in both arms were maximum in age group 31 to 50 years. The Median of age of both groups were 40 years. Sex wise distribution in both arms were maximum in male. Chi-square $=0.7659, \mathrm{df}=1, \mathrm{p}$ value $=$ 0.1907, not significant. Residence wise distribution in arm a was more in urban i.e. 16 $(55.17 \%)$ than in rural i.e. $13(44.83 \%)$. However in arm b was more in rural16 $(57.14 \%)$. Chisquare $=0.8645$, df $=1, \mathrm{p}$ value $=0.1762$, not significant. In our study, maximum involvement of the site were tongue followed by were buccal mucosa. Chi-square $=1.029, \mathrm{df}=2, \mathrm{p}$ value $=$ 0.5979 , not significant. Our study showed stages of cancer in patient of arm a was more is stage fourth i.e. $23(79.31 \%)$, followed by stage second i.e. $4(13.79 \%)$, stage third were $2(6.90 \%)$. However in arm b maximum were stage fourth 16 (57.12\%), followed by stage second 7 (25\%), stage third were 5 (17.86\%). However no patient of arm a or arm b was found in stage first. Chisquare $=3.34$, df $=2, \mathrm{p}$ value $=0.1879$, not significant. [table 1]

Table 2: shows tumor response 2 month after treatment completion

\begin{tabular}{|c|c|c|c|c|}
\hline \multirow[t]{2}{*}{$\begin{array}{l}\text { Tumor response 2month after } \\
\text { treatment completion }\end{array}$} & \multicolumn{2}{|c|}{$\begin{array}{l}\text { Crt arm } \\
(\mathrm{n}=29)\end{array}$} & \multicolumn{2}{|c|}{$\begin{array}{l}\text { Test arm } \\
(\mathrm{n}=28)\end{array}$} \\
\hline & No. & $\%$ & No. & $\%$ \\
\hline $\mathrm{Cr}$ & 19 & 65.52 & 17 & 60.71 \\
\hline $\operatorname{Pr}$ & 9 & 31.03 & 11 & 39.28 \\
\hline $\mathrm{Sd}$ & 00 & 0 & 00 & 0 \\
\hline $\mathrm{Pd}$ & 00 & 0 & 00 & 0 \\
\hline No f/u & 1 & 3.45 & 0 & 0 \\
\hline Death & 0 & 0 & 0 & 0 \\
\hline Total & 29 & 100 & 28 & 100 \\
\hline $\begin{array}{l}\text { Chi-square }=1.294 \\
\text { Degrees of freedom }=2 \\
P \text { value }=0.523 \text { (non significant) }\end{array}$ & & & & \\
\hline
\end{tabular}

One patient in arm A had last the follow up 2 month after completion of radiotherapy.

Our study showed the response of the treatment in arm A $65.52 \%$ showed complete response, $31.03 \%$ showed partial response, $0.0 \%$ showed stable disease and no one showed progressive disease in comparison to arm B $60.71 \%$ showed complete response, $39.28 \%$ showed partial response, $0.0 \%$ showed stable disease and no one showed progressive disease. Chi-square $=1.294$, $\mathrm{df}=2, \mathrm{p}$ value $=0.523$. [table 2$]$

Table: 3 shows duration of treatment and acute skin ,mucosal reaction acute skin reaction

\begin{tabular}{|c|c|c|c|c|c|}
\hline \multicolumn{2}{|l|}{ Parameter } & \multicolumn{2}{|c|}{$\begin{array}{l}\text { Crt arm } \\
(\mathrm{n}=29)\end{array}$} & \multicolumn{2}{|c|}{$\begin{array}{c}\text { Test arm } \\
(\mathrm{n}=28)\end{array}$} \\
\hline \multicolumn{2}{|l|}{ Duration of treatment } & \multicolumn{2}{|c|}{ 56-67 days } & \multicolumn{2}{|c|}{ 42-55 days } \\
\hline \multicolumn{2}{|l|}{ No.of chemotherapy } & \multicolumn{2}{|c|}{2} & \multicolumn{2}{|c|}{2} \\
\hline \multirow{3}{*}{$\begin{array}{ll}\text { Acute skin } & \text { reaction } \\
\text { (dermatitis) }\end{array}$} & 1 & 18 & $62.07 \%$ & 19 & $67.86 \%$ \\
\hline & 2 & 10 & $34.48 \%$ & 8 & $28.57 \%$ \\
\hline & 3 & 1 & $3.45 \%$ & 1 & $3.57 \%$ \\
\hline \multirow[t]{3}{*}{ Mucositis } & 1 & 20 & $68.97 \%$ & 8 & $28.57 \%$ \\
\hline & 2 & 9 & $31.03 \%$ & 19 & $67.86 \%$ \\
\hline & 3 & 0 & $0.0 \%$ & 1 & $3.57 \%$ \\
\hline Ryles tube & & & & & \\
\hline
\end{tabular}

Our study showed that the duration of the treatment in arm a was 56 to 67 days however in arm B was 42 to 55 days and number of chemotherapy 2 in Arm A and 2 in Arm B. In Arm A the dermatitis Grade 1 was $63.33 \%$, Grade 2 was $20 \%$ and Grade 3 was $16.67 \%$ in 
comparison to Arm B the Grade 1 was $43.33 \%$, Grade 2 was $40 \%$ and Grade 3 was $16.67 \%$. In Arm A the mucositis Grade 1 was $13.33 \%$, Grade $286.67 \%$ and Grade 3 was $0 \%$ in comparison to
Arm B Grade1 was 0\%, Grade2 was $60 \%$ and Grade 3 was $40 \%$. Ryle's tube in Arm A was one patient and in Arm B 3 patients. [table 3]

Tab le : $\mathbf{4}$ post treatment most commomn complain

\begin{tabular}{|l|c|c|c|c|}
\hline & \multicolumn{2}{|c|}{ Arm -A } & \multicolumn{2}{c|}{ Arm -B } \\
\hline Dryness of mouth & 13 most common 2 & $44.83 \%$ & $15 \mathrm{~m} / \mathrm{c} 2$ & $53.57 \%$ \\
\hline Loss of taste & 6 & $20.69 \%$ & 7 & $25 \%$ \\
\hline Pain & 3 & $10.34 \%$ & 5 & $17.56 \%$ \\
\hline Trismus & 3 & $10.34 \%$ & 3 & $10.71 \%$ \\
\hline Dysphasia & 2 & $6.90 \%$ & 2 & $7.14 \%$ \\
\hline Neck stiffness & 3 & $10.34 \%$ & 4 & $14.28 \%$ \\
\hline Subcutanious fibrosis & 8 & $27.59 \%$ & 9 & $32.14 \%$ \\
\hline
\end{tabular}

Our study showed post treatment most common complications in arm a was dryness of mouth $44.83 \%$, followed by subcutaneous fibrosis $27.59 \%$, loss of taste $20.69 \%$ pain, trismus and neck stiffness each is $10.34 \%$ and dysphagia $6.90 \%$ and in comparison to Arm A, Arm B dryness of mouth $53.57 \%$, followed by subcutaneous fibrosis $32.14 \%$, loss of taste $25 \%$, pain $17.56 \%$, neck Siffness $14.28 \%$, trismusn $10.71 \%$ and dysphagia $7.14 \%$ [table 4]

\section{Discussion}

Accelerated $\mathrm{rt}$ improves locoregional control in squamous cell carcinoma of head and neck, shown in different prospective randomized studies. Accelerated regimens have been shown to increase treatment-associated acute morbidity, which in severe cases might lead to an increase in late radiation effects. This study was planned with the objectives that reducing overall treatment time would negate the effect of accelerated repopulation and would result in better locoregional control. As with reduction in overall treatment time it is expected that patients will have more acute toxicity, therefore, to find out whether the patients will tolerate the new accelerated schedule and will they be able to finish the treatment as planned. The third of objective was that if the previous two objectives are met then in a busy setup like ours, the turnover on the machine will be much faster and in turn waiting list will be reduced.
Regarding loco-regional response to $\mathrm{rt}$ in our study, we observed almost equal local control both at primary and nodal site in accelerated $\mathrm{rt}$ arm as compared to conventional rt Arm

In danish head and neck cancer study group (dahanca) study(21) loco-regional tumor control improved significantly in the accelerated fractionation group compared with that in the conventional rt group (70\% vs. $60 \% 5$ years actuarial rate, $\mathrm{p}=0.0005$ ).

There was $10 \%$ statistically significant improvement in loco-regional disease control in accelerated arm. In international atomic energy agency (iaea)-acc study by overgaard et al., ${ }^{[22]}$ the 5 -year actual loco-regional control was $42 \%$ in the accelerated versus $30 \%$ in the conventional group $(\mathrm{p}=0.004)$. In our study, the statistical significance could not have reached because of the smaller sample size and shorter follow-up. But our study is certainly in accordance with dahanca trial and iaea-acc study.., the benefit of acceleration in this study was slightly similar for controlling advanced disease. Almost all treatment failures were due to insufficient locoregional tumor control.

Prolonged treatment time, for the purpose of this study was defined as completing treatment with a delay of more than 5 days. Patients who were able to complete their treatment within the stipulated time plus a 5 day allowance for logistical problems and public holidays were considered to have completed on time. Similar results were seen 
in the study by rishi a, ghoshal s et al. Where $65.52 \%$ patients in conventional arm showed complete response as compared to $60.71 \%$ patients in test arm arm and the difference was statistically insignificant. ${ }^{[23]}$ in a study by $\mathrm{k}$ shrivastava, $\mathrm{m}$ shrivastava et $\mathrm{al}^{[24]}$, out of 40 patients, 30 patients $(75 \%)$ in concomitant boost arm and 24 patients (60\%) in conventional chemoradiotherapy arm had complete response and the rest of the patients had partial response except for one patient in chemoradiotherapy arm who showed no response. In patients with residual tumor, disease recurrence, or progression of disease, salvage surgery/chemotherapy or palliative treatment was offered depending on the performance status of the individual patient, symptoms and previous treatment, after multidisciplinary tumor board meeting. The follow-up of the present study was relatively short and prevents us from commenting on the long term disease free survival, overall survival, and a more comprehensive evaluation of the late toxicities too. Another limitation of our study was the relatively smaller sample size and consequently, subgroup analyses could not be materialized.

Based on our study (though it is small), dahanca trial and iaea-acc study, we feel that six fractions per week treatment is a better option as compared to five fractions per week, especially in countries where working days are 6 in a week. The conventional schedule has been evolved in west based on their working convenience rather than based on any radiobiological or scientific evidence, as they work 5 days a week. It is also clear from the trials on accelerated $\mathrm{rt}$ delivering seven fractions per week that 7 days treatment results into unacceptable early and late toxicities. ${ }^{[25]}$ trials in which the acceleration has been more aggressive have resulted in unacceptable late morbidity if the total dose was not reduced. ${ }^{[26]}$ hence, further acceleration of treatment can also not be recommended. When concurrent chemoradiation compared to accelerated concurrent chemoradiation. The accelerated radiation offers a better compliance and toxicity profile already proved in prospective randomized trials. ${ }^{[27]}$ in our study the higher incidence of acute toxicities not much more compare to conventional radiotherapy, it could not result in treatment delays and prolonged overall radiation therapy treatment time. Overall treatment time has been observed to be one of the prime independent prognostic factors for $\mathrm{rt}$ response and hence any therapeutic advantage that could be expected from chemoradiotherapy could be nullified with the prolongation of overall treatment time. Such problems are more evident in patients who are nutritionally deprived and with poor general condition. Moreover shortening of overall treatment time will increase the turnover on treatment machine which will help to treat more number of patients in 1-year and will reduce the waiting. Hence, shortening of overall treatment time from 7 weeks to 6 weeks by use of 6 fractions per week instead of 5 fractions per week is feasible, tolerable, and results in better outcome in the patients of head and neck cancers.

\section{Conclusion}

After completing the study following conclusions have been drawn:

1- The predominantly affected age group was between 31 to 50 years which is younger as comparison to that reported in western literature.

2- Males are more commonly affected than females.

3- Most of the patients had addiction habits of bidi/cigarette smoking and tobacoo cewer with increased frequency and duration.

4- Most cases belonged to advanced stages of disease.

5- Accelerated fractionated radiotherapy that is six fractions per week is reasonable alternative to the conventional regimen that is five fractions per week, offers advantages of decreased overall treatment time, better compliance and decreased hospital stay with comparable response rate but significantly 
increased low grade acute reaction which were manageable.

\section{Reference}

1. Saranath d, khanna a. Current status of cancer burden; global and indian scenario.

2. Biomed res j. 2014;1:1-5

3. M.i. Saunders et al. Head and neck cancer: altered fractionation schedules. The oncologist. 1999;4:11-16.

4. Krstevska v. Radiotherapy and chemotherapy in locally advanced head and neck squamous cell carcinoma. J buon. 2009; 14:361-73.

5. Fu kk. Biological basis for the interaction of chemotherapeutic agents and radiation therapy. Cancer. 55:2123.1985.

6. Maciejewski $\mathrm{b}$, preuss-bayer $\mathrm{g}$, trott $\mathrm{kr}$. The influence of the number of fractions and overall treatment time on local control and late complication rate in squamous cell carcinoma of the larynx. Int $\mathrm{j}$ radiat oncol biol phys. 1983;9:321-328.

7. Withers, h.r., taylor, j.m., maciejewski, b. The hazard of accelerated tumor clonogen repopulation during radiotherapy. Acta oncol. 1988;27:131-146.

8. Pinto, 1.h., canary, p.c., araujo, c.m., bacelar, s.c., souhami, 1. Prospective randomized trial comparing hyperfractionated versus conventional radiotherapy in stages iii and iv oropharyngeal carcinoma. Int $\mathrm{j}$ radiat oncol biol phys. 1991;21:557-562.

9. Horiot, j.c., le fur, r., n'guyen, t. Et al, hyperfractionation versus conventional fractionation in oropharyngeal carcinoma: final analysis of a randomized trial of the eortc cooperative group of radiotherapy. Radiother oncol. 1992;25:231-241.

10. Fu, k.k., pajak, t.f., trotti, a. Et al, a radiation therapy oncology group (rtog) phase iii randomized study to compare hyperfractionation and two variants of accelerated fractionation to standard fractionation radiotherapy for head and neck squamous cell carcinomas: first report of rtog 9003.int $\mathrm{j}$ radiat oncol biol phys. 2000;48:7-16.

11. Overgaard, j., hansen, h.s., specht, 1. Et al, five compared with six fractions per week of conventional radiotherapy of squamouscell carcinoma of head and neck: dahanca 6 and 7 randomised controlled trial. Lancet. 2003;362:933-940.

12. Bourhis, j.s.n., overgaard, j., ang, k.k. Et al, conventional vs modified fractionated radiotherapy. Meta-analysis of radiotherapy in head and necksquamous cell carcinoma: a meta-analysis based on individual patient data. Int $\mathrm{j}$ radiat oncol biol phys. 2004;s190-s191.

13. Keane t, fyles a, o' sullivan b, et al: the effect of treatment duration on local control of squamous carcinoma of the tonsil and carcinoma of the cervix. Seminradiat oncol 2:26-28, 1992

14. Overgaard j, sand hansen h, overgaard m, et al: importance of overall treatment time for the outcome of radiotherapy in head and neck carcinoma: experience from the danish head and neck cancer study, in kogelnik h, sedlmayer f (eds):

15. Sixth international meeting on progress in radio-oncology, pp 743-752. Bologna, italy, monduzzi editore, 1998.

16. Skladowski k, maciejewski b, golen $\mathrm{m}$, et al: randomized clinical trial on 7daycontinuous accelerated irradiation (cair) of head and neck cancer \pm report on 3 -year tumour control and normal tissue toxicity. Radiother oncol 55:101-110, 2000.

17. Jackson s, weir 1 , hay $\mathrm{j}$, et al: a randomised trial of accelerated vs conventional radiotherapy in head and $\backslash$ neck cancer. Radiother oncol 43:39-46, 1997.

18. Withers hr. Biologic basis for altered fractionation schemes. Cancer. 1985;55(9 suppl):2086-9 
19. Nguyen ln, ang kk. Radiotherapy for cancer of the head and neck: altered fractionation regimens. Lancet oncol. 2002;3:693-701.

20. Bernier j, bentzen sm. Altered fractionation and combined radiochemotherapy approaches: pioneering new opportunities in head and neck oncology. Eur j cancer. 2003;39:560-71.

21. Fu kk, pajak tf, trotti a, jones cu, spencer sa, phillips tl, et al. A radiation therapy oncology group (rtog) phase iii randomized study to compare hyperfractionation and two variants of accelerated fractionation to standard fractionation radiotherapy for head and neck squamous cell carcinomas: first report of rtog 9003. Int $\mathrm{j}$ radiat oncol biol phys. 2000;48:7-16.

22. Hansen o, overgaard j, hansen hs, overgaard m, höyer $m$, jörgensen ke, et al. Importance of overall treatment time for the outcome of radiotherapy of advanced head and neck carcinoma: dependency on tumor differentiation. Radiother oncol. 1997;43:47-51.

23. Sanghvi ld, jayant k, pakhale ss. Tobacco use and cancer in india. World smoking health 1980;5:4-10. (published by anet. Cancer society)

24. Rao dn, desai pb, ganesh b. Alcohol as an additional risk factor in laryngopharyngeal cancer in mumbai - a case-control study. Cancer detect prev 1999;23:37-44

25. Rishi a, ghoshal s, verma r, oinamas,patilvm, mohinder $\mathrm{r}$ et al. Comparison of concomitant boost radiotherapy against concurrent chemoradiation in locally advanced oropharyngeal cancers: a phase iii randomised trial; radiotherapy and oncology. 2013;107:317-24.
26. Srivastava $\mathrm{k}$, srivastava $\mathrm{m}$. Concomitant boost radiotherapy vs conventional radiotherapy in advanced oral cavity and oropharyngeal cancers. Indian journal of radiology and imaging. 2001;11:12730.14 .

27. Kapil u, singh p, bahadur s, dwivedi sn, singh $\mathrm{r}$, shukla $\mathrm{n}$. Assessment of risk factors in laryngeal cancer in india: a casecontrol study. Asian pac $\mathrm{j}$ cancer prev 2005;6:202-7.

28. Sapkota a, gajalakshmi v, jetly dh, roy chowdhury s, dikshit rp, brennan $\mathrm{p}$, et al. Smokeless tobacco and increased risk of hypopharyngeal and laryngeal cancers: a multicentric case-control study from india. Int j cancer 2007;121:1793-8.

29. Roy chowdhury s, roy chowdhury g, sen u. Assessment of awareness level on tobacco and smoking habits as risk factors for cancer among lung and laryngeal cancer patients in kolkata - a case control study. Asian pac $\mathrm{j}$ cancer. 